\title{
Modelos do sistema cardiovascular para apoio ao diagnóstico
}

\author{
André Leite, \\ UFPE - Departamento de Estatística \\ E-mail: leite@de.ufpe.br \\ Luís H. de Santana \\ UFPE - Departamento de Matemática \\ E-mail: desantananlh@gmail.com
}

\author{
Fernando Menezes Campello de Souza \\ UFPE - Departamento de Eletrônica e Sistemas \\ E-mail: fmcs@hotlink.com.br
}

\begin{abstract}
Resumo: Estuda-se a dinâmica do sistema cardiovascular à luz de modelos matemáticos de sistemas dinâmicos. O leito arterial é representado por uma equação diferencial ordinária de primeira ordem (chamado modelo de Windkessel de dois elementos), de onde foram elaborados os parâmetros. O desenvolvimento de novos marcadores teve como paradigma básico a elaboração de procedimentos de apoio ao diagnóstico das disfunções do sistema cardiovascular que pudessem fazer uso de exames complementares simples, como a aferição da pressão arterial e da freqüência cardíaca. Buscou-se aumentar o potencial destes testes na prática clínica. A Ecocardiografia, o exame de Hölter-24h, a Monitorização Ambulatorial da Pressão Arterial de 24 horas, o Eletrocardiograma, a Série Bioquímica, entre outros exames, foram usados, ora como padrões ouro, ora como marcadores das diversas disfunções do sistema, entre estas a hipertrofia ventricular esquerda, a diabetes e arritmias. Os resultados foram vindicados por intermédio de uma base de dados com 3.035 (três mil e trinta e cinco) indivíduos.
\end{abstract}

Palavras-chave: engenharia cardiovascular, apoio ao diagnóstico, hipertensão arterial

\section{Introdução}

O sistema cardiovascular (SCV) vem recebendo uma atenção crescente, pois as doenças do sistema cardiovascular foram as causas de mais de 17 milhões de mortes no mundo, em 2008 [6]. No Brasil, esse número ultrapassou 300 mil [6]. Mais de 300 fatores de risco foram associados com as disfunções do sistema cardiovascular. Os principais fatores de risco satisfazem a três critérios: uma alta prevalência em muitas populações; impacto independente no risco da doença cardíaca; e o tratamento e controle destes fatores resulta numa diminuição do risco.

Nos países em desenvolvimento, no mínimo um terço de todas as doenças cardiovasculares são associadas a cinco fatores de risco: tabagismo, uso de álcool, hipertensão arterial, colesterol alto e obesidade [6, parte 2]. Alguns dos principais fatores de risco podem ser modificados, pode-se prevenir, tratar ou controlá-los.

Neste contexto, tem-se os seguintes objetivos:

- Estudar a dinâmica do sistema cardiovascular, via modelos matemáticos.

- Elaborar parâmetros (ou marcadores) que sejam representativos do sistema circulatório.

- Propor modelos de apoio ao diagnóstico. 


\section{Modelo do leito arterial - modelo de Windkessel}

O Modelo de Windkessel é usado para descrever a carga (pressão) a que o coração é submetido enquanto bombeia o sangue através do leito arterial, assim como a relação entre a pressão sangüínea e o fluxo sangüíneo na aorta. ${ }^{1}$ Caracterizar a carga do sistema arterial no coração em termos de parâmetros provenientes do modelo de Windkessel, tais como complacência e resistência periférica, é de importância, por exemplo, para a quantificação de efeitos de drogas vasodilatadoras ou vasoconstritoras.

Assumindo-se que a relação da pressão do ar e volume do ar na câmara é constante; e que o fluxo do fluído nos tubos que conectam a câmara à bomba segue a lei de Poiseuille e é proporcional a pressão do fluído, a seguinte equação diferencial é encontrada para relacionar o fluxo de líquido e pressão:

$$
I(t)=\frac{P(t)}{R}+C \frac{d P(t)}{d t}
$$

em que $I(t)$ é o fluxo de água que sai da bomba em função do tempo, medido em unidade de volume por tempo, $P(t)$ é a pressão em função do tempo medida em unidade de força por área, $C$ é a relação constante da pressão do ar e volume do ar; e $R$ é a constante de proporcionalidade fluxo-pressão.

Em termos fisiológicos, $I(t)$ é o fluxo de sangue do coração para a aorta, medido em, e.g., centímetros cúbicos por segundo $\left(\mathrm{cm}^{3} / \mathrm{s}\right) ; P(t)$ é a pressão sangüínea na aorta em milímetros de mercúrio $(\mathrm{mm} \mathrm{Hg}) ; C$ é a complacência arterial da aorta em unidade de centímetros cúbicos por milímetro de mercúrio $\left(\mathrm{cm}^{3} / \mathrm{mm} \mathrm{Hg}\right)$; e $R$ é a resistência periférica da circulação sistêmica em unidade de milímetros de mercúrio por centímetro cúbico por segundo $\left(\mathrm{mm} \mathrm{Hg} \cdot \mathrm{s} / \mathrm{cm}^{3}\right)$.

Durante a diástole, quando não existe fluxo do coração para a aorta, $I(t)=0$, e dessa maneira tem-se a seguinte solução para a pressão, $P(t)$, na aorta durante a diástole:

$$
P(t)=P\left(t_{d}\right) \cdot e^{-\frac{1}{\tau}\left(t-t_{d}\right)},
$$

em que $t_{d}$ é o tempo onde inicia diástole e $P\left(t_{d}\right)$ é a pressão sangüínea na aorta em $t_{d}$.

Durante a sístole, o coração bombeia um certo volume $V_{0}$, chamado de volume de ejeção, para o leito arterial. Assumindo-se, por simplificação, um tempo de sístole nulo, i.e., todo o volume é ejetado instantaneamente no leito arterial, tem-se a seguinte solução para o modelo de Windkessel de 2 elementos:

$$
P(t)=P(0) \cdot e^{-\frac{t}{\tau}}
$$

em que $P(0)$ é equivalente a pressão sistólica $\left(P_{s}\right)$ e $P(T)$ é equivalente a pressão diastólica $\left(P_{d}\right)$, em que $T$ é a duração do ciclo cardíaco. Note-se que $\tau$ é o produto da resistência periférica pela complacência da aorta, podendo ser estimado a partir de $P_{s}, P_{d}$ e T, i.e.,

$$
\tau=\frac{T}{\left[\log P_{s}-\log P_{d}\right]}
$$

A pressão arterial média, conhecida na literatura médica por PAM e aqui notada por $\bar{P}_{\tau}$, é calculada a partir do modelo:

$$
\begin{aligned}
\bar{P}_{\tau} & =\frac{1}{T} \int_{0}^{T} P(t) d t \\
& =\frac{\tau}{T}\left(P_{s}-P_{d}\right) \quad \Rightarrow \\
\bar{P}_{\tau} & =\frac{P_{s}-P_{d}}{\log P_{s}-\log P_{d}}
\end{aligned}
$$

Na Figura 1 apresenta-se uma comparação entre dados reais da pressão arterial e o resultado do modelo de Windkessel.

\footnotetext{
${ }^{1}$ Restringe-se aqui ao modelo da circulação sistêmica; o mesmo modelo poderá ser utilizado para a circulação pulmonar. Desprezam-se as pressões do lado venoso.
} 


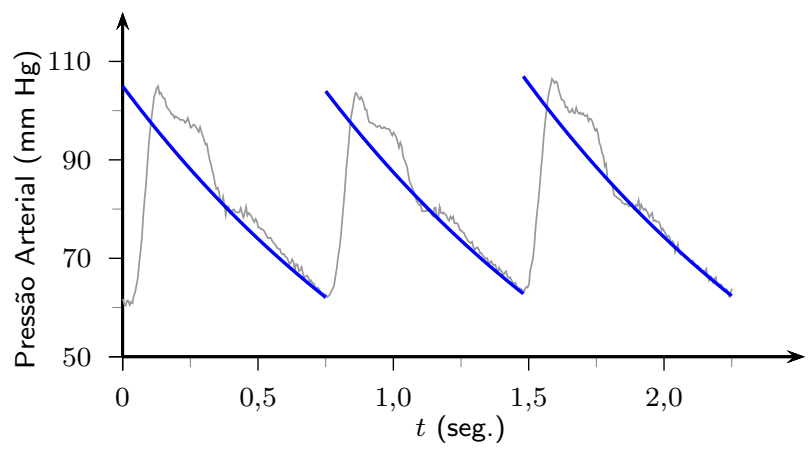

Figura 1: Contorno da curva de pressão arterial.

Seja $\bar{P}_{\tau}$ a pressão arterial média do modelo de Windkessel, calculada pela Expressão 5 , e $\bar{P}$ a pressão arterial média calculada a partir de uma integração numérica dos dados. Para os dados mostrados na Figura 1, obteve-se uma correlação (R de Spearman) significativa de 0,975.

Note-se que, a partir de medidas simples $\left(P_{s}, P_{d}\right.$ e $\left.F_{c}\right)$, obtidas diretamente no consultório médico com instrumentos corriqueiros, é possível estimar o parâmetro $\tau$ ( $c f$. expressão 4$)$, que nada mais é do que o produto da complacência (essencialmente das grandes artérias) pela resistência periférica (essencialmente nas arteríolas). O parâmetro $\tau$ varia ao longo do ciclo circadiano, devido principalmente aos ajustes provenientes do barorreflexo [5]. Entretanto, ao contrário da pressão sistólica, diastólica e freqüência cardíaca, o parâmetro $\tau$ é reprodutível [1].

O parâmetro $\tau$ é um indicador do funcionamento dinâmico do SCV. Ele controla a forma da curva pressórica arterial. Supondo que a complacência das grandes artérias é constante ${ }^{2}$, dois mecanismos de controle (ou malhas de realimentação) podem alterar o valor de $\tau$. Primo, o mecanismo de auto-regulação (controle local), pois, de acordo com as necessidades metabólicas nos tecidos, o diâmetro das arteríolas e a condição dos esfíncteres pré-capilares podem se alterar. Secondo, o ramo simpático do sistema nervoso autonômico, por intermédio do barorreflexo [5], controla o tonus muscular nas artérias menores, podendo também, alterar a resistência do sistema arterial. O balanço entre estes dois sistemas de controles ainda é pouco entendito [5]; a principal dificuldade é a escassez de dados, ou melhor, a falta de dados adequados, como, e.g., a aquisição simultânea do pressão arterial, eletrocardiograma, respiração, $O_{2}$ no sangue arterial, $O_{2}$ no sangue venoso e fonocardiograma.

\section{Modelos top-down da dinâmica da pressão arterial}

Sempre no paradigma da simplicidade, tentar-se-á modelar a dinâmica da pressão arterial de modo a levar em consideração o tempo de sístole, suposto nulo na seção anterior.

A sístole pode ser dividida em duas fases, contração isovolumétrica e ejeção. Quando o ciclo cardíaco é iniciado, o ventrículo esquerdo começa a contrair-se, alterando a pressão interna, embora, como a válvula de acesso ao sistema arterial ainda esteja fechada, não haja alteração de volume, essa é chamada fase isovolumétrica.

Quando a pressão no ventrículo atinge a pressão na aorta (nesse instante chamada pressão diastólica), a válvula é então aberta e dá-se inicio a fase de ejeção. A pressão continua subindo até atingir o ponto de máximo. Este tempo de ejeção, entre a abertura da válvula até a pressão atingir seu máximo, está relacionado tanto com a força de contração e freqüência cardíaca, parâmetros intrínsecos ao coração, quanto com o sistema arterial, pois depende basicamente da complacência e resistência do leito arterial.

\footnotetext{
${ }^{2}$ Sabe-se que a complacência se altera com a idade, indivíduos idosos possuem uma complacência menor. Todavia, essa alteração se processa de forma muito mais lenta que a dinâmica do SCV, tornando razoável a hipótese de uma complacência constante.
} 


\subsection{Modelo de ajuste triangular}

Seja $\alpha_{\triangle}$ a relação entre o tempo de ejeção e o período do ciclo ( $c f$. Figura 2). Para estimar essa proporção, faz-se a hipótese de que o ângulo de subida (entre a curva da pressão e o eixo das pressões) é igual ao ângulo de descida, formado a partir de uma reta horizontal partindo da pressão sistólica e a curva da pressão.

Dessa maneira, obtém-se a seguinte relação:

$$
\frac{\alpha_{\triangle} T}{P_{p}}=\frac{P_{p}}{\left(1-\alpha_{\triangle}\right) T}
$$

onde $P_{p}$ é a pressão de pulso e $T$ é período do ciclo cardíaco. Resolvendo essa equação para $\alpha_{\triangle}$, chega-se à seguinte expressão:

$$
\alpha_{\triangle}=\frac{1}{2}-\frac{1}{2 T} \sqrt{T^{2}-4 P_{p}^{2}}
$$

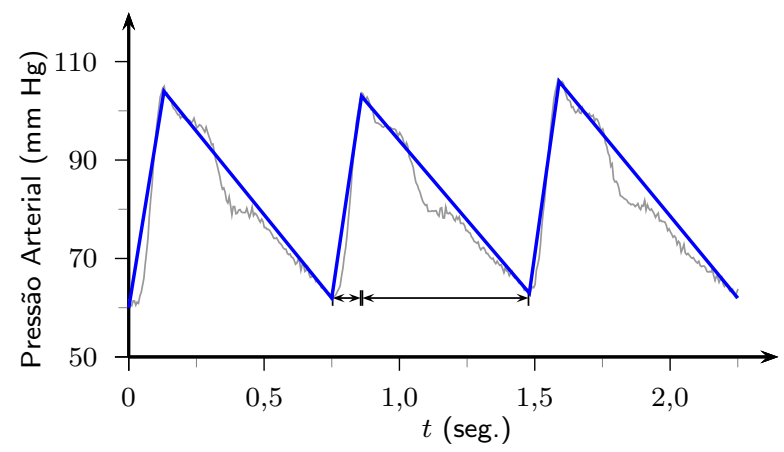

Figura 2: Contorno da curva de pressão arterial.

A pressão arterial média, $\bar{P}_{\triangle}$, nesse caso, vai ser dada pela média aritmética da pressão sistólica e diastólica. Obteve-se uma correlação (R de Spearman) significativa de 0,969.

\subsection{Modelo de ajuste polinomial}

Considere-se agora, uma função polinomial da seguinte maneira

$$
P(t)=k_{1} \cdot t \cdot(T-t)^{n}+k_{2},
$$

quer-se ajustar à esta equação um ciclo da pressão arterial $(0<t<T), c f$. Figura 3.

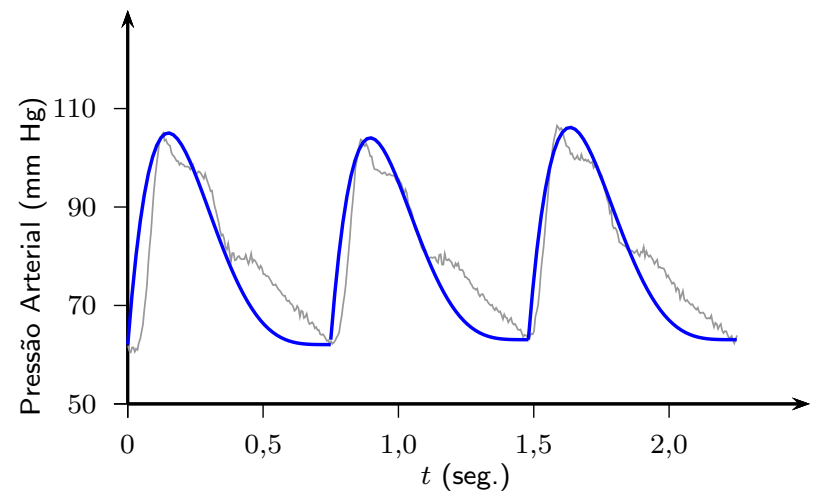

Figura 3: Contorno da curva de pressão arterial e ajuste polinomial. 
Tem-se primeiro que calcular $k_{1}, k_{2}$ e $n$ em função das variáveis $P_{s}, P_{d}$ e $T$. Note que, para $t=0$ ou $t=T, P(T)=P(0)=k_{2}$ e portanto $k_{2}=P_{d}$. O ponto de máximo dessa função deve ser igual a $P_{s}$, tem-se então:

$$
\begin{aligned}
\frac{d P(t)}{d t} & =k_{1}(T-t)^{n}-k_{1} \operatorname{tn}(T-t)^{n-1} ; \\
0 & =k_{1}\left(T-t^{*}\right)^{n}-k_{1} t^{*} n\left(T-t^{*}\right)^{n-1} \Rightarrow \\
t^{*}+n t^{*} & =T
\end{aligned}
$$

e portanto, o ponto de máximo ocorre em

$$
t^{*}=\frac{T}{n+1}
$$

Para encontrar $k_{1}$, basta igualar o valor da função no ponto de máximo à $P_{s}$ :

$$
\begin{aligned}
P\left(t^{*}\right) & =P_{s} \\
k_{1} t^{*}\left(T-t^{*}\right)^{n}+P_{d} & =P_{s} \\
k_{1} n^{n}\left(\frac{T}{n+1}\right)^{n+1} & =P_{p}
\end{aligned}
$$

Tem-se então

$$
k_{1}=P_{p}\left(\frac{n+1}{T}\right)^{n+1} n^{-n}
$$

Substituindo $k_{1}$ e $k_{2}$ na função 8 :

$$
P(t)=P_{p} \cdot\left(\frac{n+1}{T}\right)^{n+1} \cdot n^{-n} \cdot t \cdot(T-t)^{n}+P_{d}
$$

Pode-se calcular a pressão arterial média, $\bar{P}_{n}$, usando a seguinte expressão:

$$
\bar{P}_{n}=P_{d}+\frac{(n+1)^{n+1}}{(n+2) n^{n}} \cdot P_{p}
$$

Note-se que o tempo de ejeção, tempo entre o inicio do ciclo e pico da pressão arterial, é exatamente $t^{*}$, tempo onde ocorre o máximo da pressão. A relação entre o tempo de ejeção e o período, $\alpha_{n}$, é dada por

$$
\alpha_{n}=\frac{t^{*}}{T}=\frac{1}{n+1}
$$

Ao contrário dos parâmetros anteriores $\left(\tau\right.$ e $\left.\alpha_{\triangle}\right)$, esse novo parâmetro $\alpha_{n}$, não pode ser estimado apenas com uma medida de $P_{s}, P_{d}$ e $T$. Necessita-se da pressão arterial média. A partir dos dados disponibilizados pelo $\operatorname{InCor}^{3}$, pode-se estimar $\alpha_{n}$ por intermédio de uma regressão não linear ${ }^{4}$, com base na expressão 12 . A Tabela 1 mostra o resultado da regressão.

Tabela 1: Resultado da estimação do parâmetro $n$.

\begin{tabular}{r|r}
$\hat{n}$ & 4,926 \\
Erro padrão & 0,0097 \\
Significância $(p)$ & 0 \\
Variância explicada & $96 \%$ \\
\hline
\end{tabular}

Tem-se então, $\alpha_{\hat{n}} \approx 0,17$, ou seja, o tempo de ejeção foi $17 \%$ do tempo total do ciclo cardíaco. Trata-se de um valor nos limites da normalidade [4]. A Figura 4 mostra o diagrama de 


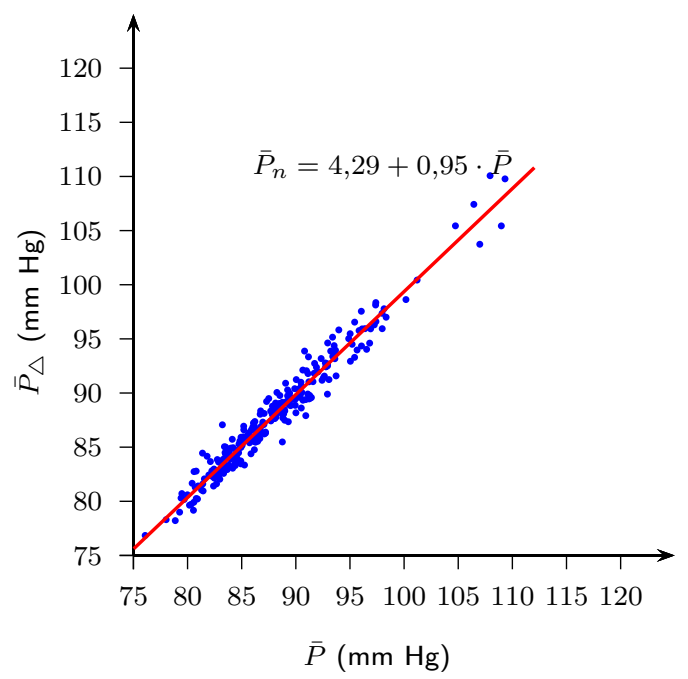

Figura 4: Relação entre a média calculada e a média estimada no ajuste polinomial.

dispersão entre a pressão arterial média e a pressão arterial média estimada a partir do modelo. A correlação ( $\mathrm{R}$ de Spearman) é significativa com valor 0,975 .

Na Tabela 2, apresenta-se o resultado da estimação de $n$ com dados de exame de MAPA- $24 \mathrm{~h}^{5}$, provenientes da base de dados do Professor Hilton Chaves $[3,2]$.

Tabela 2: Resultado da estimação do parâmetro $n$ em um exame de MAPA-24h.

\begin{tabular}{r|r}
$\hat{n}$ & 6,376 \\
Erro padrão & 0,0395 \\
Significância $(p)$ & 0 \\
Variância explicada & $94 \%$ \\
\hline
\end{tabular}

\section{Resultados e Conclusões}

Os parâmetros propostos, obtidos a partir de modelos matemáticos do sistema cardiovascular, mostraram-se reprodutíveis e correlacionados com diversos marcadores do estado de saúde desse sistema. Abriu-se então a perspectiva para o uso desses parâmetros, isoladamente ou em conjunto, no apoio ao diagnóstico e acompanhamento das condições do sistema cardiovascular. A Tabela 3 mostra quais os parâmetros que estão correlacionados com quais as condições do sistema cardiovascular. Os resultados dos diversos procedimentos estatísticos, i.e., a vindicação dos modelos, encontram-se em [5].

Há que se explorar melhor essas relações trabalhando-se com uma base de dados correspondente a uma amostra bem maior.

Note-se que o parâmetro $\tau$, do SCV, está mais ligado ao leito arterial. Os parâmetros $\alpha_{\triangle}$, $\alpha_{n}, t^{*}$, e os derivados destes, também são do SCV, mas têm ligação maior com o coração.

\footnotetext{
${ }^{3}$ Instituto do Coração da Faculdade de Medicina da Universidade de São Paulo.

${ }^{4}$ Utilizou-se o algoritmo Rosenbrock Pattern Search.

${ }^{5}$ Monitorização Ambulatorial da Pressão Arterial.
} 
Tabela 3: Relação entre os parâmetros e o sistema cardiovascular.

\begin{tabular}{l|c|c|c}
\hline & $\alpha_{\triangle}$ & $\alpha_{n}$ & $t^{*}$ \\
\hline HVE & $\times$ & $\times$ & \\
Glicemia & $\times$ & & \\
Parâmetros elétricos & $\times$ & $\times$ & $\times$ \\
Parâmetros mecânicos & $\times$ & $\times$ & \\
Sensibilidade do barorreceptor & $\times$ & & \\
Classificação da hipertensão & $\times$ & $\times$ & \\
Série bioquímica & $\times$ & $\times$ & $\times$ \\
Hormônios & & $\times$ & $\times$ \\
\hline
\end{tabular}

\section{Agradecimentos}

Ao Dr. Luciano Drager e a Dr. Patrícia Lessa, pesquisadores do InCor/SP, que forneceram dados para estimação de parâmetros do sistema cardiovascular.

\section{Referências}

[1] F. M. Campello de Souza. O apoio ao diagnóstico médico: o que se pode fazer com um tensiômetro e um relógio. Recife: Vade Mecum, 2004.

[2] H. Chaves. Monitorização ambulatorial da pressão arterial (MAPA-24h): sua reprodutibilidade e importância na construção de modelos para estimar a hipertrofia cardíaca. Tese de Doutorado, Universidade de São Paulo, São Paulo, 2000.

[3] L. Chaves Rêgo e F. M. Campello de Souza. Improved estimation of left ventricular hypertrophy. IEEE Engineering in Medicine and Biology, v. 18, n. 1, p. 66 - 68, 2002.

[4] A. T. Guyton Tratado de fisiologia médica. 6. ed. Rio de Janeiro: Editora Guanabara, 1981.

[5] A. LeIte. Sobre a dinâmica do sistema cardiovascular. Dissertação, Universidade Federal de Pernambuco, Recife, 2005.

[6] WHO. Global atlas on cardiovascular disease prevention and control. Genova: World Health Organization, 2011. 